\title{
Modelos Pseudo Polinomiais para o Problema do Empacotamento Colorido *
}

\author{
Y. G. F. Borges ${ }^{1}$, R. C. S. Schouery ${ }^{1}$. \\ ${ }^{1}$ Instituto de Computação - Universidade Estadual de Campinas (UNICAMP) \\ Av. Albert Einstein, 1251 - 13083-852 - Campinas - SP - Brasil \\ \{glebbyo,rafael\}@ic.unicamp.br
}

\begin{abstract}
Resumo. O Problema do Empacotamento Colorido consiste em empacotar um conjunto de itens, cada um com um tamanho e cor, na menor quantidade possivel de recipientes de uma dada capacidade, de forma que não haja dois itens de uma mesma cor em sequência em nenhum recipiente. Propomos formulações pseudo polinomiais de Programação Inteira que resolvem instâncias geradas aleatoriamente com até 500 itens e 15 cores em segundos.
\end{abstract}

\begin{abstract}
The Colored Bin Packing Problem consists in packing a set of items, each with a size and a color, into the minimum amount of bins of a given capacity, such that no two items from the same color appear consecutively in the same bin. We propose pseudo-polynomial Integer Programming formulations that solve randomly generated instances of up to 500 items and 15 colors in a matter of seconds.
\end{abstract}

\section{Introdução}

Problemas de Corte e Empacotamento são clássicos na literatura e são estudados pela comunidade científica desde os anos trinta (Kantorovich, 1939). Múltiplas variantes destes problemas foram estudadas devido sua alta aplicabilidade em diferentes indústrias.

Neste trabalho, tratamos especificamente do Problema do Empacotamento Colorido (CBPP, do inglês Colored Bin Packing Problem), no qual cada item, além de um tamanho também possui uma cor, e queremos evitar que dois itens de uma mesma cor apareçam lado a lado em qualquer recipiente. Este problema foi proposto por Balogh et al. (2013), que consideravam uma variante com apenas duas cores. Balogh et al. (2015) apresentaram um AFPTAS e um APTAS, que por sua vez apresenta uma razão de aproximação absoluta de 3/2, para esta variante. Posteriormente, Böhm et al. (2018) propuseram análises de vários algoritmos online para a variante com mais de duas cores, incluindo um algoritmo 3/2-competitivo. O CBPP é definido formalmente a seguir.

Problema do Empacotamento Colorido (CBPP). São dados um conjunto de itens $\mathcal{I}=\{1, \ldots, m\}$, cada item $u \in \mathcal{I}$ com tamanho inteiro $l_{u}>0$ e cor $c_{u} \in[Q] \mathrm{e}$ recipientes de capacidade $L$. O objetivo é encontrar uma partição de $\mathcal{I}$ de forma que a soma dos tamanhos dos itens em cada parte é menor ou igual a $L$ e dois itens de uma mesma cor não apareçam lado a lado em nenhuma das partes.

*Pesquisa financiada pela FAPESP, processos \#2015/11937-9, \#2016/01860-1 e \#2016/23552-7, e CNPq, processos \#425340/2016-3, \#308689/2017-8 e \#144257/2019-0. O presente trabalho foi realizado com apoio da Coordenação de Aperfeiçoamento de Pessoal de Nível Superior - Brasil (CAPES) - Código de Financiamento 001 
Consideramos que $\left\{\mathcal{I}_{1}, \mathcal{I}_{2}, \ldots, \mathcal{I}_{Q}\right\}$ é uma partição de $\mathcal{I}$ em cores, isto é, $u \in \mathcal{I}_{q} \Longleftrightarrow c_{u}=q$. Além disso, itens com um mesmo tamanho e cor são agrupados em um único item com um valor de demanda inteiro $d_{u}>0$ indicando sua multiplicidade.

Por fim, note que o clássico Problema do Empacotamento (BPP, do inglês Bin Packing Problem), é um caso particular do CBPP onde cada item possui uma cor diferente.

\section{Modelos Pseudo Polinomiais para o CBPP}

Modelos pseudo polinomiais são aqueles em que o número de variáveis ou restrições cresce de maneira pseudo polinomial de acordo com a entrada do problema. Geralmente, estes modelos apresentam limitantes mais justos quando comparados com modelos compactos. Várias formulações pseudo polinomiais foram propostas para o BPP, destacandose o de Fluxo em Arcos de Valério De Carvalho (1999). Nesta seção, apresentamos novos modelos para o CBPP baseados nesta ideia.

\subsection{Fluxo em Arcos}

Considere um digrafo $G^{\prime}=\left(V^{\prime}, A^{\prime}\right)$. O conjunto de vértices $V^{\prime}$ é a união entre um único vértice origem 0 e, para cada cor $q \in[Q]$, um conjunto de vértices $V_{q}^{\prime}$ enumerados de 1 até $L$, que representam os pontos de empacotamento para um item desta cor em um certo recipiente. O conjunto de arcos $A^{\prime}$ é definido da seguinte forma: para todo vértice $i \in V_{q}^{\prime}$, existe um arco-item conectando $i$ com algum $j \in V_{q^{\prime}}^{\prime}$ se $q \neq q^{\prime}$ e existe um item de tamanho $j-i$ em $\mathcal{I}_{q^{\prime}}$; existe um arco-item conectando 0 com todo vértice $j \in V_{q^{\prime}}^{\prime}$ tal que exista um item de tamanho $j$ em $\mathcal{I}_{q^{\prime}}$ para todo $q^{\prime} \in[Q]$; para todo vértice $i \in V^{\prime} \backslash\{0\}$ existe um arco-perda conectando $i$ com o $L$-ésimo vértice de alguma cor.

Podemos utilizar o modelo de fluxo em arcos de Valério De Carvalho (1999), construído sob o grafo $G^{\prime}$, para encontrar soluções para o CBPP. Tais soluções sempre respeitarão as restrições de cores, uma vez que de acordo com a definição de $G^{\prime}$, não existirão caminhos que passem por dois vértices de uma mesma cor consecutivamente. Uma vez que o modelo encontra o menor número de caminhos que cobre os arcos de todos os itens em demanda, sua solução será também ótima para o CBPP.

\subsection{Fluxo em Arcos com Alternância de Cores}

Nesta seção propomos um novo modelo para o CBPP, ao qual nos referimos como Fluxo em Arcos com Alternância de Cores, que utiliza um grafo muito menor. Para isto, considere o multi-grafo direcionado $G=(V, A)$ definido da seguinte forma. O conjunto $V$ é formado por um vértice origem 0 , e uma sequência de vértices enumerados de 1 até $L$, que representam os pontos de empacotamento para os itens em um recipiente. O conjunto de $\operatorname{arcos} A$ é definido da seguinte forma: para todo vértice $i \in V \backslash\{0, L\}$ existe um arcoperda conectando $i$ a $L$; para cada cor $q \in[Q]$, existe um conjunto de arcos-item $A_{q} \subseteq A$ que conectam vértices $i$ e $j$ para todo $(i, j)$ tal que exista um item de tamanho $j-i$ em $\mathcal{I}_{q}$.

Considerando este grafo, propomos a seguinte formulação, na qual $x$ é um conjunto de variáveis inteiras que indicam a quantidade de fluxo passando por cada arco e $z$ indica a quantidade de fluxo passando pelos arcos que partem da origem 0 . 
minimize $z$

$$
\begin{aligned}
& \text { sujeito a } \quad \sum_{(i, j) \in A} x_{i j}-\sum_{(j, k) \in A} x_{j k}= \begin{cases}-z & \text { para } j=0, \\
0 & \text { para } j=1, \ldots, L-1, \\
z & \text { para } j=L,\end{cases} \\
& \sum_{(i, j) \in A_{q}} x_{i j}-\sum_{(j, k) \in A \backslash A_{q}} x_{j k} \leq 0 \quad \begin{aligned}
\text { para } j & =1, \ldots, L-1, \\
q & =1, \ldots, Q,
\end{aligned} \\
& \sum_{(i, j) \in A_{q}: j=i+l_{u}} x_{i j}=d_{u} \quad \text { para } u \in \mathcal{I}_{q}, q=1, \ldots, Q \\
& z, x_{i j} \in \mathbb{Z}^{+} \quad \forall(i, j) \in A,
\end{aligned}
$$

As restrições (2) garantem a conservação de fluxo em qualquer vértice em $V$. As restrições (3) forçam que a quantidade de fluxo passando por arcos de cor $q$ que chegam em um vértice $j$ não será maior que a quantidade de fluxo passando por arcos de uma cor diferente de $q$ saindo de $j$. As restrições (4) garantem que as demandas para todos os itens serão satisfeitas, e por fim, as restrições (5) garantem a integralidade das soluções.

Teorema 1. A formulação de Fluxo em Arcos com Alternância de Cores (1)-(5) modela corretamente o Problema do Empacotamento Colorido (CBPP).

\subsection{Reduções dos Grafos}

O tamanho dos modelos pseudo polinomiais baseados em fluxo em arcos cresce com a quantidade de vértices e arestas em seus respectivos grafos. Utilizamos o conceito de padrões normais (também conhecido como dissecações canônicas), proposto por Herz (1972) e Christofides e Whitlock (1977), para reduzir o tamanho dos grafos sem perder soluções ótimas. A ideia principal por trás deste conceito é de que qualquer padrão de empacotamento viável pode ter seus itens movidos ao máximo para esquerda de forma que toda a perda esteja no fim do recipiente.

\section{Experimentos Computacionais}

Os modelos foram implementados utilizando a linguagem de programação C++ e o resolvedor de Programação Linear Inteira Gurobi 8.2 (Gurobi Optimization, 2020). Executamos ambos os modelos para cada uma das instâncias propostas com um limite de tempo de 1800 segundos. Os experimentos foram executados em modo single-thread em um Intel(R) Xeon(R) CPU E5-2630 de 2.20 GHz, 10 núcleos e 64 GB de RAM.

\subsection{Instâncias}

O conjunto de instâncias proposto é caracterizado por cinco parâmetros principais: quantidade de itens $m$, tamanho dos recipientes $L$, quantidade de cores $Q$, tamanho do menor item $l_{\min }$ e tamanho do maior item $l_{\max }$. Consideramos $m \in\{100,300,500\}$, $L \in\{200,400,500\}, Q \in\{2,7,15\}, l_{\min }=0.1 L$ e $l_{\text {max }}=0.8 L$. Os tamanhos dos itens foram gerados a partir de uma distribuição uniforme entre $l_{\min }$ e $l_{\max }$, enquanto as cores foram distribuídas uniformemente garantindo que haja pelo menos um item de cada cor. Foram geradas 5 instâncias para cada combinação dos parâmetros descritos, totalizando 135 instâncias. 


\subsection{Resultados}

Em nossos resultados, observamos que o modelo de Fluxo em Arcos conseguiu resolver um total de 134 instâncias na otimalidade, levando em média 56.17 segundos por instância. O segundo modelo, Fluxo em Arcos com Alterância de Cores, resolveu todas as 135 instâncias na otimalidade, levando em média 6.62 segundos por instância.

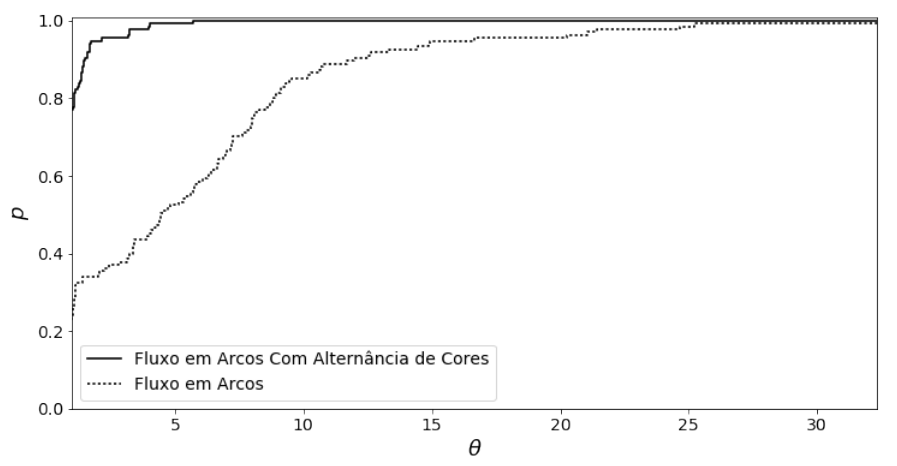

Figura 1. Performance Profile considerando tempo de solução dos métodos

A Figura 1 mostra o Performance Profile (Dolan e Moré, 2002) do tempo de solução para os modelos. Cada algoritmo resolve uma instância com um determinado custo de tempo, que é então escalado em relação ao custo do melhor algoritmo. Assim, temos no eixo $\theta$ o fator desta escala. No eixo $\rho$, temos a porcentagem das instâncias consideradas. Desta forma, conseguimos observar que o modelo com Alternância de Cores foi o mais rápido em aproximadamente $80 \%$ das instâncias, e quando não foi o melhor levou, no pior caso, até 5 vezes o tempo do outro método. Com isso podemos concluir que, para este tipo de instâncias, o modelo com Alternância de Cores é melhor que o modelo de Fluxo em Arcos. Entretanto, é necessário um estudo experimental mais robusto, com um conjunto de instâncias mais abrangente, para se fazer conclusões mais fortes.

\section{Referências}

Balogh, J., Békési, J., Dósa, G., Epstein, L., Kellerer, H., Levin, A., e Tuza, Z. (2015). Offline black and white bin packing. Theoretical Computer Science, 596:92-101.

Balogh, J., Békési, J., Dosa, G., Kellerer, H., e Tuza, Z. (2013). Black and white bin packing. In Proceedings of the $10^{\text {th }}$ International Workshop on Approximation and Online Algorithms, pages 131-144.

Böhm, M., Dósa, G., Epstein, L., Sgall, J., e Veselý, P. (2018). Colored bin packing: Online algorithms and lower bounds. Algorithmica, 80(1):155-184.

Christofides, N. e Whitlock, C. (1977). An algorithm for two-dimensional cutting problems. Operations Research, 25(1):30-44.

Dolan, E. D. e Moré, J. J. (2002). Benchmarking optimization software with performance profiles. Mathematical programming, 91(2):201-213.

Gurobi Optimization, L. (2020). Gurobi optimizer reference manual.

Herz, J. (1972). Recursive computational procedure for two-dimensional stock cutting. IBM Journal of Research and Development, 16(5):462-469.

Kantorovich, L. V. (1939). The mathematical method of production planning and organization. Management Science, 6:363-422.

Valério De Carvalho, J. (1999). Exact solution of bin-packing problems using column generation and branch-and-bound. Annals of Operations Research, 86:629-659. 